\title{
The indirect search for dark matter with the ANTARES neutrino telescope
}

Christoph Tönnis, on behalf of the ANTARES Collab.

IFIC - Instituto de Física Corpuscular,

Universitat de València-CSIC,

E-46100 Valencia, Spain

E-mail: ctoennisdific.uv.es,

The indirect search for dark matter is a topic of utmost interest in neutrino telescopes. The ANTARES detector is located at the bottom of the Mediterranean Sea $40 \mathrm{~km}$ off the southern french coast. Results of the indirect searches for dark matter self-annihilation signals from different potential sources, including the Sun and the Galactic Center, produced with different analysis methods are presented. The specific advantages of neutrino telescopes in general and of ANTARES in particular will be explained. As an example, the indirect search for Dark Matter towards the Sun performed by neutrino telescopes currently leads to the best sensitivities and limits on the spin-dependent WIMP-nucleon cross section with respect to existing direct detection experiments.

The 34th International Cosmic Ray Conference,

30 July- 6 August, 2015

The Hague, The Netherlands 


\section{Introduction}

One of the concepts in the indirect search for dark matter is to look for annihilations of WIMPS in massive celestial objects. WIMPS can accumulate in these celestial objects due to gravitational capture or due to the formation of dark matter halos in the early universe [1]. The annihilations of those WIMPS can produce standard model particles which can produce photons and neutrinos in secondary processes. These neutrinos and photons can then be detected in different experiments.

In this paper, the results of the ANTARES neutrino telescope [2] on the searches for neutrinos from the center of the Milky Way, of the Sun and on dwarf galaxies are presented. In the following, neutrinos stands for both neutrino and antineutrinos. The search for dark matter in the Earth is presented in another contribution [3].

In the case of extended sources, as our Galaxy, galaxies and galaxy clusters as possible sources the so called J-Factor has to be calculated. The J-Factor is necessary to relate the neutrino signal flux to the thermal averaged annihilation cross section, which is a parameter that depends on the actual dark matter model employed and is customarily used to express the sensitivities and limits of the experiments, both direct and indirect, for the sake of comparison. The J-Factor is the squared dark matter density integrated along the line of sight, and can be calculated with the formula:

$$
\mathrm{J}(\theta)=\int_{0}^{1_{\max }} \frac{\rho_{\mathrm{DM}}^{2}\left(\sqrt{\mathrm{R}_{\mathrm{SC}}^{2}-21 \mathrm{R}_{\mathrm{SC}} \cos (\theta)+\mathrm{l}^{2}}\right)}{\mathrm{R}_{\mathrm{SC}} \rho_{\mathrm{SC}, \mathrm{DM}}^{2}} \mathrm{dl}
$$

$\mathrm{R}_{\mathrm{SC}}$ is the scaling radius of the halo and $\rho_{\mathrm{SC}, \mathrm{DM}}$ is the scaling density. The J-Factor then relates

$$
\frac{\mathrm{d} \phi_{v}}{\mathrm{dE}}=\frac{<\sigma \mathrm{v}>}{2} \mathrm{~J}_{\Delta \Omega} \frac{\mathrm{R}_{\mathrm{SC}} \rho_{\mathrm{SC}}^{2}}{4 \pi \mathrm{m}_{\chi}^{2}} \frac{\mathrm{dN}_{v}}{\mathrm{dE}}
$$

where $\mathrm{J}_{\Delta \Omega}$ is the $\mathrm{J}$-Factor integrated over the observation window $\Delta \Omega, \mathrm{m}_{\chi}$ is the WIMP mass and $\frac{\mathrm{dN} \mathrm{N}_{V}}{\mathrm{dE}}$ is the expected signal neutrino spectrum. The dark matter halo profile $\rho_{\mathrm{DM}}$ is fitted to measurement data as for example the distribution of rotational velocities of stars in the galaxy in question. For the profile of the Dark Matter halo the NFW function is used [4]:

$$
\rho(\mathrm{r})=\frac{\rho_{\mathrm{s}}}{\left(\mathrm{r} / \mathrm{r}_{\mathrm{s}}\right)\left(1+\mathrm{r} / \mathrm{r}_{\mathrm{s}}\right)^{2}}
$$

with $\mathrm{r}_{\mathrm{s}}=21.7 \mathrm{kpc}$. The normalisation of the profile density, $\rho_{\mathrm{s}}$, is computed by fixing the dark matter density at the Sun's position $\rho\left(\mathrm{r}_{\text {Sun }}=8.5 \mathrm{kpc}\right)=0.4 \mathrm{GeV} \cdot \mathrm{cm}^{-3}$.

In the case of the Sun a different approach has to be chosen to calculate sensitivities and limits in terms of dark matter model parameters. In this case, it is assumed that there is an equilibrium between the gravitational capture of WIMPS by their scattering with the solar plasma and the annihilation of WIMPS in the Sun. If the average number of neutrinos per WIMP annihilation is known, the total neutrino flux can be related to the total annihilation rate in the Sun, which is proportional to the capture rate. This capture rate can be expressed as [5]:

$$
\mathrm{C}_{\mathrm{s}}=3.35 \frac{1}{\mathrm{~s}}\left(\frac{\rho_{\mathrm{loc}}}{0,3 \frac{\mathrm{GeV}}{\mathrm{cm}^{3}}}\right)\left(\frac{270 \frac{\mathrm{km}}{\mathrm{s}}}{\mathrm{v}_{\mathrm{rms}}}\right)^{3}\left(\frac{\sigma_{\mathrm{H}, \mathrm{sd}}+\sigma_{\mathrm{H}, \mathrm{si}}+0,07 \sigma_{\mathrm{He}, \mathrm{si}}}{10^{-6} \mathrm{pb}}\right)\left(\frac{100 \mathrm{GeV}}{\mathrm{m}_{\chi}}\right)^{2}
$$


The different $\sigma$ are the spin-dependent and spin-independent cross section for the scattering of WIMPS with hydrogen and helium, $v_{\text {rms }}$ is the root mean squared velocity of the WIMPS in the galactic halo at the Sun position and $\rho_{\text {loc }}$ is the local dark matter density.

In the analyses presented here some representative annihilation channels of the WIMPs have been chosen in order to stay model independent. For each annihilation channel a $100 \%$ branching ratio for the WIMP annihilation directly into a specific pair of standard model particles is assumed. The following channels have been used:

$$
\mathrm{WIMP}+\mathrm{WIMP} \rightarrow b+\bar{b}, W^{+}+W^{-}, \tau^{+}+\tau^{-}, \mu^{+}+\mu^{-}, v+\bar{v}
$$

The $v \bar{v}$ and $\mu^{+} \mu^{-}$channel have not been considered for the search for WIMP annihilations in the Sun. The $\tau^{+} \tau^{-}$channel is most commonly used as a benchmark for comparisons between experiments [6]. For the Sun additional effects have to be taken into account. These effects are the absorption of neutrinos and the regeneration of tau neutrinos in the solar plasma [7, 8].

The ANTARES detector has obtained different limits on the flux of neutrinos from astrophysical objects. In Section 2 the result from an "unbinned" search method from the direction of the Sun is presented. In Section 3 a "binned" method is used for the searches for an excess of neutrinos from the direction of the Galactic center and from dwarf galaxies. In the "unbinned" method, the sensitivities and upper limits are constructed using a likelihood function. This likelihood function can be written as:

$$
\log _{10}\left(\mathrm{~L}\left(\mathrm{n}_{\mathrm{s}}\right)\right)=\sum_{\mathrm{i}=1}^{\mathrm{N}_{\text {tot }}} \log _{10}\left(\mathrm{n}_{\mathrm{s}} \mathrm{S}\left(\psi_{\mathrm{i}}, \mathrm{p}_{\mathrm{i}}, \mathrm{q}_{\mathrm{i}}\right)+\mathrm{N}_{\mathrm{tot}} \mathrm{B}\left(\psi_{\mathrm{i}}, \mathrm{p}_{\mathrm{i}}, \mathrm{q}_{\mathrm{i}}\right)\right)-\mathrm{n}_{\mathrm{s}}-\mathrm{N}_{\text {tot }}
$$

$\mathrm{N}_{\text {tot }}$ is the total number of reconstructed events, $n_{s}$ is the supposed number of signal events, $\psi_{\mathrm{i}}$ is the angular position of the $\mathrm{i}_{\text {th }}$ event, $\mathrm{p}_{\mathrm{i}}$ and $q_{i}$ are additional event parameters like the reconstruction quality or the estimated neutrino energy. $\mathrm{S}$ represents the ANTARES point spread function (PSF) for the signal and $\mathrm{B}$ is a function that represents the behavior of the background.

This likelihood function is then used to analyse pseudo experiments. A pseudo experiment is a sky map filled with simulated background events, generated from a background estimate and a given number of fake signal events, using the PSF and the signal statistics. For each pseudo experiment the likelihood function is optimized with respect to $n_{s}$. A parameter called the test statistics (TS) is then calculated as:

$$
\mathrm{TS}=\log _{10}\left(\frac{\mathrm{L}\left(\mathrm{n}_{\mathrm{S}}\right)}{\mathrm{L}(0)}\right)
$$

The sensitivities in terms of detected signal events $\mu_{90 \%}$ are calculated from the overlap of the distribution of TS values for different numbers of inserted fake signal events. Upper limits on the number of signal events are then calculated comparing the TS value of the actual data to the TS distributions of pseudo experiments.

The sensitivities and limits are then converted to neutrino fluxes using a quantity referred to as acceptance. The acceptance is defined as: 


$$
\operatorname{Acc}\left(\mathrm{m}_{\text {WIMP }}, \mathrm{Ch}\right)=\left.\int_{\mathrm{E}_{\mathrm{th}}}^{\mathrm{m}_{\mathrm{WIMP}}} \mathrm{A}_{\mathrm{eff}}\left(\mathrm{E}_{v_{\mu}}\right) \frac{\mathrm{dN}_{v_{\mu}}}{\mathrm{dE}_{v_{\mu}}}\right|_{\text {Det,Ch }} \mathrm{dE}_{v_{\mu}}+\left.\int_{\mathrm{E}_{\mathrm{th}}}^{\mathrm{m}_{\mathrm{WIMP}}} \mathrm{A}_{\mathrm{eff}}\left(\mathrm{E}_{\bar{v}_{\mu}}\right) \frac{\mathrm{dN}_{\bar{v}_{\mu}}}{\mathrm{dE}_{\bar{v}_{\mu}}}\right|_{\text {Det, Ch }} \mathrm{dE}_{\bar{v}_{\mu}}
$$

where $A_{\text {eff }}\left(E_{v_{\mu}}\right)$ is the effective area for the muon neutrino energy $E_{v_{\mu}}$ or muon antineutrino energy $\mathrm{E}_{v_{\bar{\mu}}},\left.\frac{\mathrm{dN}_{v_{\mu}}}{\mathrm{dE}_{v}}\right|_{\text {Det,Ch }}$ is the signal neutrino spectrum at the position of the detector for one particular annihilation channel $\mathrm{Ch}$ listed in equation $1.5, \mathrm{E}_{\mathrm{th}}$ is the energy threshold of the detector and $\mathrm{m}_{\text {WIMP }}$ is the WIMP mass. The effective area, which is the size of the detector assuming a $100 \%$ detection efficiency, is calculated from the Monte Carlo simulation. The $90 \%$ C.L. limits and sensitivities on the fluxes are then calculated by:

$$
\bar{\Phi}_{v_{\mu}+\bar{v}_{\mu}, 90 \%}=\frac{\bar{\mu}_{v_{\mu}+\bar{v}_{\mu}, 90 \%}\left(\mathrm{~m}_{\mathrm{WIMP}}\right)}{\operatorname{Acc}\left(\mathrm{m}_{\mathrm{WIMP}}\right) \cdot \mathrm{T}_{\text {live }}}
$$

where $\bar{\mu}_{v_{\mu}+\bar{v}_{\mu}, 90 \%}$ is the $90 \%$ C.l. sensitivity or limit and $\mathrm{T}_{\text {live }}$ is the total live time of the detector.

\section{Indirect search for Dark Matter towards the Sun}

An indirect search for DM towards the Sun has been performed using data collected during 2007 and 2012. No excess of data has been observed in the direction of the Sun. Limits have been calculated in terms of muon neutrino fluxes and spin-dependent WIMP-nucleon scattering cross sections, which can be seen in figure 1 and 2, respectively. As can be seen in the flux limits the loosest cross section limits stem from the $b \bar{b}$ channel which is the softest of the three channels used in that analysis. The $\tau^{+} \tau^{-}$and the $\mathrm{W}^{+} \mathrm{W}^{-}$channel lead to harder neutrino spectra and give very similar flux limits.

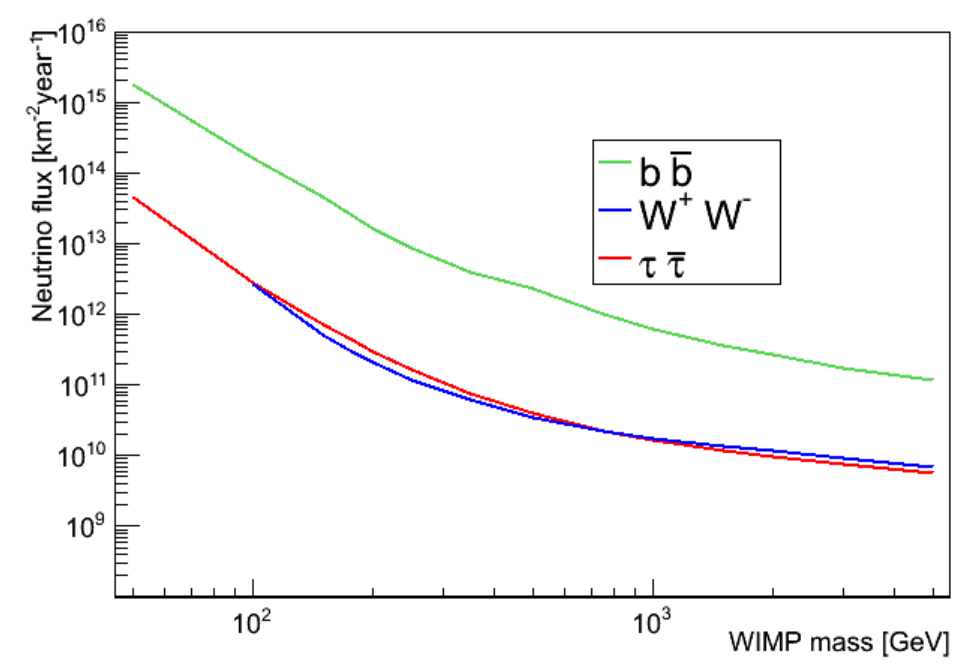

Figure 1: $90 \%$ upper limits on the muon neutrino flux originating from self-annihilations of dark matter inside the Sun as a function of the WIMP mass obtained by the analysis of the data recorded by ANTARES between 2007 and 2012 
The spin-dependent WIMP-proton scattering cross section limits depend on the scattering of WIMPS with hydrogen in the Sun, whilst the spin-independent cross section limits depend on the scattering with helium. Since hydrogen is much more abundant in the Sun indirect searches are more sensitive to the spin-dependent scattering cross section and can surpass even direct detection experiments. Direct detection experiments, as Xenon 100 or LUX, are definitively more competitive for the spin-independent cross section. The ANTARES limits are more stringent than those of Ice Cube at higher masses (hundreds of $\mathrm{GeV}$ ), although the instrumented volume of IceCube is significantly larger. This is due to the fact that IceCube limits are dependent on the performance of its central Deep Core [9]. In addition, the angular resolution in the measurement of hundreds of $\mathrm{GeV}$ neutrinos is better in water than in ice.

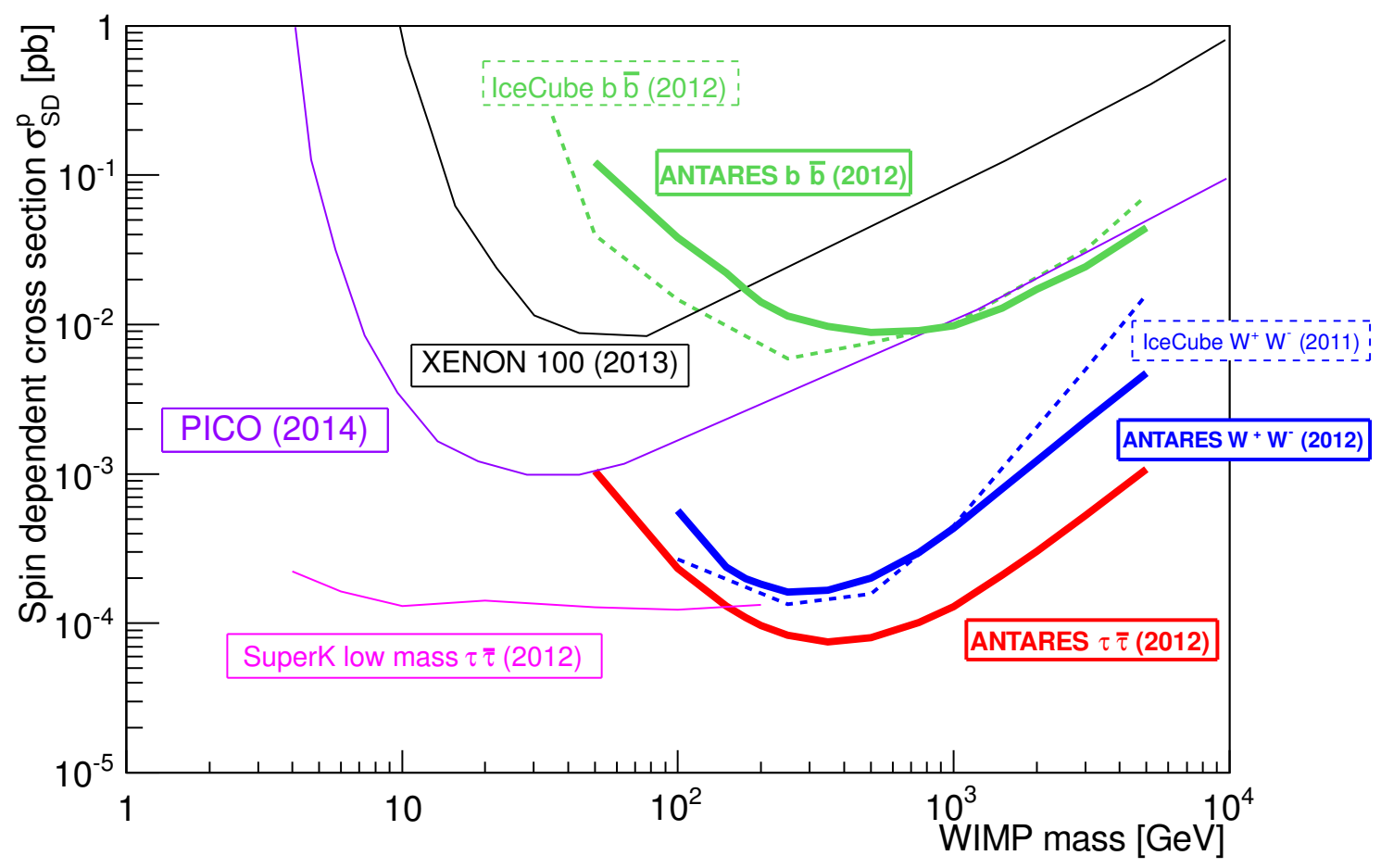

Figure 2: 90\% C.L. upper limits on the spin-dependent WIMP-proton scattering cross section as a function of the WIMP mass. Limits of other experiments are shown $[9,11,12,13]$.

\section{Indirect search for Dark Matter towards the Galactic Center and dwarf galaxies}

For the indirect search for Dark Matter towards the Galactic Center a "binned" analysis method has been used. This method calculates the amount of events within a cone around of the source and compare this to a background estimate. The sensitivities and limits are then calculated from the amount of events observed within the cone compared to those expected for the background. The size of this cone is optimized using background estimates and the sensitivities generated with it. No significant excess over the expected background has been found in the ANTARES data 
recorded between 2007 and 2012 and therefore exclusion limits have been calculated. In figure 3 this exclusion limit in terms of neutrino signal fluxes in the direction of the GC is shown. As previously, the least stringent limit comes from the $\mathrm{b} \overline{\mathrm{b}}$ channel, the $v_{\mu} v_{\bar{\mu}}$ channel lead to the most stringent limits. These limits are then converted to thermal averaged cross sections using J-Factors calculated assuming a NFW profile in equation 1.6.

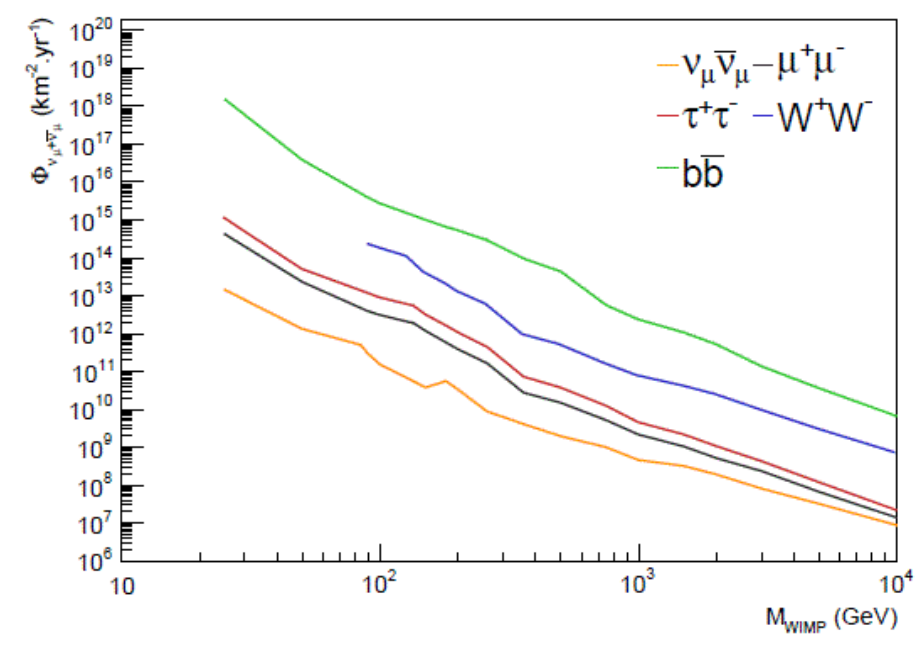

Figure 3: $90 \%$ C.L. upper limits on the neutrino flux, $\Phi_{v_{\mu}+\bar{v}_{\mu}}$, originating from self-annihilation of dark matter in the direction of the GC, as a function of the WIMP mass in the range $25 \mathrm{GeV} \leq \mathrm{M}_{\mathrm{WIMP}} \leq 10 \mathrm{TeV}$ for the self-annihilation channels (from top to bottom) WIMPWIMP $\rightarrow$ $\mathrm{b} \overline{\mathrm{b}}$ (green), $\mathrm{W}^{+} \mathrm{W}^{-}$(blue), $\tau^{+} \tau^{-}$(red), $\mu^{+} \mu^{-}$(darkgrey), $\nu_{\mu} \bar{v}_{\mu}$ (orange).

In figure 4, the $90 \%$ C.L upper limit for the velocity averaged self-annihilation cross-section $<\mathrm{sv}>$ obtained by ANTARES is compared to that of other experiments. The $\tau^{+} \tau^{-}$channel has been chosen for the comparison. The original limit obtained by the IceCube experiment [10] looking at the Galactic Center uses different halo parameters. Therefore in figure $4 \mathrm{a}$ factor has been applied to the IceCube limits. This factor is the ratio of the integrated J-Factor used in the IceCube analysis to a J-Factor calculated using the halo parameters defined in section 1. It is worth to notice that the limits from ANTARES reject at 90\% C.L. the interpretation of the PAMELA excess as a signal of leptophilic dark matter, if the constrains from HESS and Fermi-LAT [14] are also applied.

A similar analysis has been performed looking for a neutrino signal originating from dark matter annihilation in several dwarf spheroidal galaxies. No excess of events towards those objects has been found in the data recorded by ANTARES between 2007 and 2012. In order to derive an upper limit on the WIMP velocity averaged self-annihilation cross section, the signal of the 3 dwarf galaxies presenting the largest J-Factor and visibilities have been stacked. The resulting limit has also been included in figure 4 . 


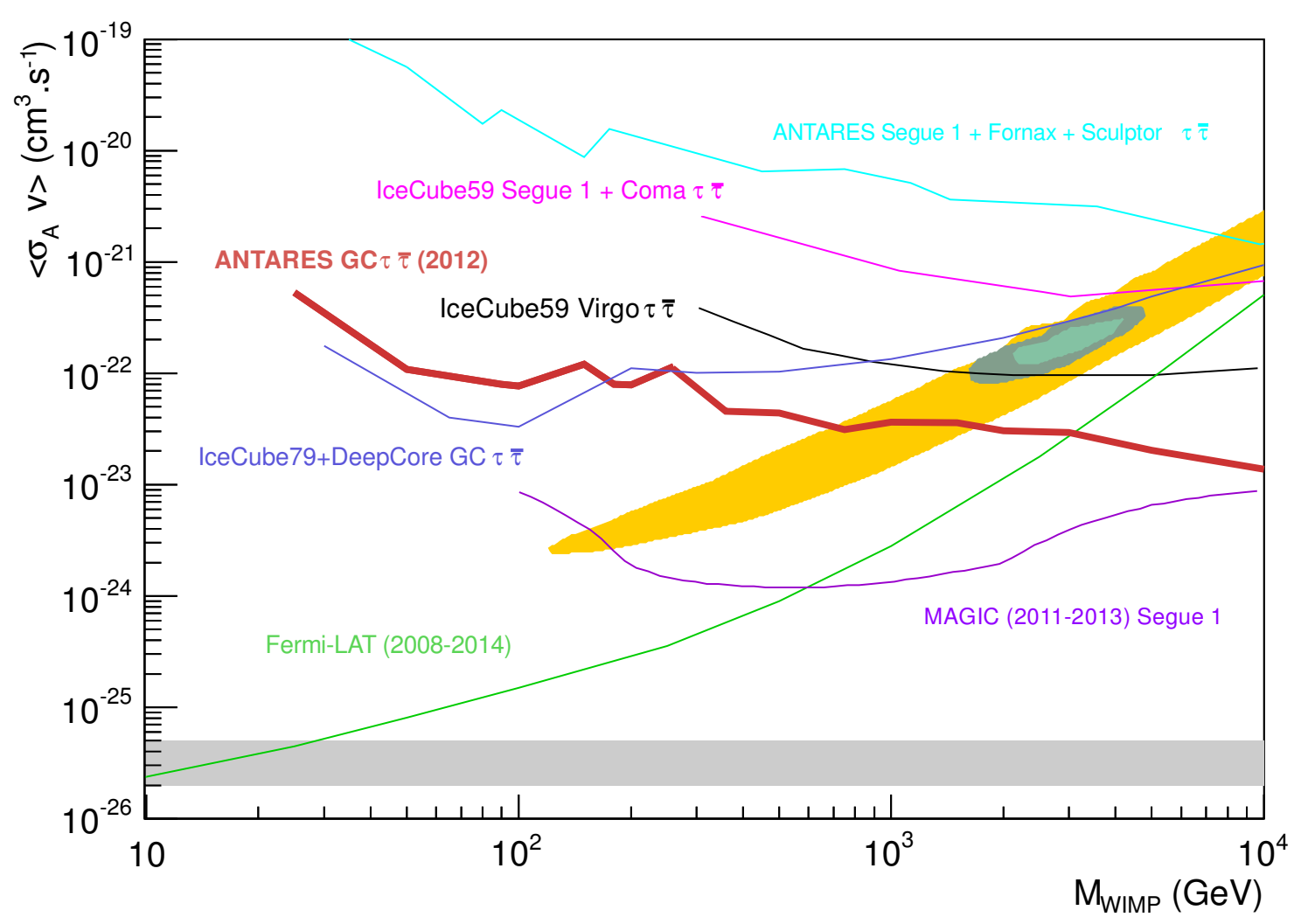

Figure 4: $90 \%$ C.L. upper limits on the WIMP velocity averaged self-annihilation cross-section, $\left\langle\sigma_{\mathrm{A}} \mathrm{v}\right\rangle$, as a function of the WIMP mass in the range $10 \mathrm{GeV} \leq \mathrm{M}_{\text {WIMP }} \leq 10 \mathrm{TeV}$. In this plot the IceCube limit for the Galactic Center is corrected with a constant factor for the different J-Factors used in the analysis. Limits of various experiments are shown [10, 15, 16, 17, 18]. The allowed region of [14] arising from the PAMELA positron excess is also shown.

\section{Conclusion}

As one can see the different searches for dark matter with the ANTARES neutrino telescope lead to limits, that can compete with the results of comparable experiments. Concerning especially the analysis for the Galactic Center the ANTARES limits are currently the most stringent limits from all neutrino telescopes, once the difference between the halo models used in the analyses is taken into account. Future improvements on this analysis, including the use of more advanced analysis methods, the inclusion of more recent data from ANTARES and a complementary analysis searching for neutrinos from WIMP annihilations in galaxy clusters are currently planned or in progress.

\section{References}

[1] A. Gould, Direct And Indirect Capture Of Wimps By The Earth, Astrophys. J. 328, 919 (1988); T. K. Gaisser, G. Steigman, and S. Tilav, Limits on Cold Dark Matter Candidates from Deep Underground Detectors, Phys. Rev. D 34, 2206 (1986); J. Silk et al., The Photino, the Sun and High-Energy 
Neutrinos, Phys. Rev. Lett. 55, 257 (1985); W. H. Press and D. N. Spergel, Capture by the sun of a galactic population of weakly interacting, massive particles, Astrophys. J. 296, 679 (1985).

[2] J.A. Aguilar et al. ANTARES: the first undersea neutrino telescope, arXiv 1104.1607v1.

[3] A. Gleixner, this conference.

[4] A. Charbonnier, C. Combet, D. Maurin, CLUMPY: A code for $\gamma$-ray signals from dark matter structures, Comp. Phys. Comm. 183, 656 (2012).

[5] A. Gould Cosmological Density of WIMPS from Solar and Terrestrial Annihilations, Astrophys. J. IASSNS-AST-91-34 1991.

[6] ANTARES Collaboration, Search of Dark Matter Annihilation in the Galactic Centre using the ANTARES Neutrino Telescope, arXiv:1505.04866v1.

[7] M. Boudard, M. Cirelli, G. Giesen, P. Salati, A fussy revisitation of antiprotons as a tool for Dark Matter searches, arXiv:1412.5696.

[8] Mattias Blennow, Joakim Edsjo, Tommy Ohlsson Neutrinos from WIMP Annihilations Obtained Using a Full Three-Flavor Monte Carlo Approach, arXiv:0709.3898.

[9] The IceCube collaboration, M. G. Aartsen et al., Search for dark matter annihilations in the Sun with the 79-string IceCube detector, Phys. Rev. Lett. 110, 131302.

[10] The IceCube collaboration, Search for Dark Matter Annihilation in the Galactic Center with IceCube-79, arXiv:1505.07259.

[11] C. Amole et al., Dark Matter Search Results from the PICO-2L $C_{3} F_{8}$ Bubble Chamber, Phys. Rev. Lett. 114, 231302 (2015).

[12] Super-Kamiokande Collaboration, Search for Neutrinos from Annihilation of Captured Low-Mass Dark Matter Particles in the Sun by Super-Kamiokande, Phys. Rev. PRL 114, 141301 (2015).

[13] The XENON100 Collaboration, Limits on spin-dependent WIMP-nucleon cross sections from 225 live days of XENON100 data, arXiv:1301.6620v2.

[14] P. Meade, M. Papucci, A. Strumia, T. Volansky, Dark Matter Interpretations of the Electron/Positron Excesses after FERMI, Nucl. Phys. B831, 178-203 (2010).

[15] IceCube Collaboration, M.G. Aartsen et al., The IceCube Neutrino Observatory Part IV: Searches for Dark Matter and Exotic Particles, 33rd International Cosmic Ray Conference, Rio de Janeiro, 2013 [astro-ph/1309.7007].

[16] IceCube Collaboration, M.G. Aartsen et al., IceCube Search for Dark Matter Annihilation in nearby Galaxies and Galaxy Clusters, Phys. Rev. D88 122001, (2013)

[17] Fermi-LAT Collaboration, M. Ackermann et al., Dark Matter Constraints from Observations of 25 Milky Way Satellite Galaxies with the Fermi Large Area Telescope, Phys. Rev. D 89, 042001 (2014).

[18] MAGIC Collaboration, J. Aleksić et al., Optimised dark matter searches in deep observations of Segue 1 with MAGIC, JCAP02, 008 (2014). 